\title{
Diagnóstico del potencial turístico para el diseño de senderos ecoturísticos. Caso Bosque Protector Cerro Blanco, Guayas, Ecuador
}

\section{Diagnosis of the tourist potential for the design of ecotourism trails. The Case of the Cerro Blanco Protected Forest, Guayas, Ecuador}

Diana Delgado Campuzano ${ }^{1}$, Roberto Herrera Anangono², Amada Zambrano Rodriguez ${ }^{3}$, Gabriela Torres Jara ${ }^{3}$, Jazmin Peñafiel León ${ }^{3}$, María Jose Ortíz ${ }^{3}$

Resumen: El objetivo principal del Bosque Protector Cerro Blanco (BPCB) es "Proteger y rehabilitar una muestra representativa de importancia nacional de la región del bosque seco tropical, fomentando la comprensión, apreciación y deleite público para no destruirlo y guardarlo para las generaciones presentes y futuras, tanto de ecuatorianos como visitantes internacionales" (Horstman, 1998). Para analizar la sustentabilidad de la Zona de Recreación al Aire Libre del BPCB es necesario conocer el diagnóstico de los diferentes elementos que conforman el paisaje, tales como las condiciones climáticas, las características del relieve, la potencialidad del suelo, la estructura de la vegetación, y la fauna. Dicho análisis permite identificar los procesos o cambios que en un momento dado pueden alterar el funcionamiento de un sistema ambiental. La implementación del diseño de los nuevos senderos para el BPCB implica la elaboración de todos aquellos accesorios o construcciones necesarias para el buen desarrollo del mismo. El objetivo final es evaluar alternativas para implementarlas en el lugar considerando la normativa técnica, ambiental y turística.

Palabras clave: Gestión ambiental, procedimiento general, uso responsable de recursos.
Abstract: The main objective of the Cerro Blanco Protected Forest (BPCB in Spanish) is "To protect and rehabilitate a nationally important representative sample of the tropical dry forest region, promoting public understanding, appreciation and enjoyment so as not to destroy it and to maintain it for present and future generations, both Ecuadorian as well as international visitors" (Horstman, 1998). In order to analyze the sustainability of the Outdoor Recreation Area of the $B P C B$, it is necessary to understand the diagnosis of the different elements that comprise the landscape, such as the climate conditions, the characteristics of the terrain, soil potential, vegetation structure and the fauna. This analysis makes it possible to identify the processes or changes that can alter the functioning of an environmental system at a certain time. The implementation of the design of the new trails for the $B P C B$ involves preparing all those accessories or constructions needed for their proper development. The ultimate aim is to assess alternatives to implement them in place, considering technical, environmental and tourist regulations.

Keywords: Environmental management, general procedure, responsible use of resources.

(Presentado: 10 de febrero de 2017. Aceptado: 29 de marzo de 2017)

\footnotetext{
${ }^{1}$ M.Sc. Universidad Estatal de Milagro, Milagro, Guayas, Ecuador. E-mail: dianavaleriadcayahoo.com

${ }^{2}$ M.Sc. Universidad Tecnica Estatal de Quevedo, Quevedo, Ecuador.

${ }^{3}$ M.Sc. Universidad Estatal de Milagro, Milagro, Guayas, Ecuador.
} 


\section{INTRODUCCIÓN}

La conservación y protección de la naturaleza es un tema que ha despertado gran interés a nivel mundial, y la creación de áreas protegidas ha permitido resguardar los recursos naturales y culturales de muchos sitios que los poseen en mayor número (Boo, 1989). El objetivo principal del Bosque Protector Cerro Blanco (BPCB) es "Proteger y rehabilitar una muestra representativa de importancia nacional de la región del bosque seco tropical, fomentando la comprensión, apreciación y deleite público para no destruirlo y guardarlo para las generaciones presentes y futuras, tanto de ecuatorianos como visitantes internacionales" (Horstman, 1998). Para analizar la sustentabilidad de la Zona de Recreación al Aire Libre (ZRAL) del BPCB es necesario conocer el diagnóstico de los diferentes elementos que conforman el paisaje, tales como las condiciones climáticas, las características del relieve, la potencialidad del suelo, la estructura de la vegetación y la fauna. A través de dicho análisis se logra identificar los procesos o cambios que en un momento dado pueden alterar el funcionamiento de un sistema ambiental.

La implementación dentro de los parámetros de la investigación del diseño de los nuevos senderos para el BPCB busca elaborar todos aquellos accesorios y construcciones necesarias para el buen desarrollo del mismo, lo cual considera como implementación; esto es buscar la necesidad en base a parámetros técnicos, dar una o varias alternativas para suplir dicha necesidad, implementarlas en el lugar correspondiente tomando en consideración la normativa técnica, ambiental y turística.

\section{MATERIALES Y METODOLOGÍA}

El BPCB se encuentra en la parroquia Tarqui, cantón Guayaquil, provincia del Guayas (Figuta 1). Está ubicado geográficamente a una Latitud: $2^{\circ} 7^{\prime} \mathrm{S}$, Longitud: $80^{\circ} 5^{\prime} \mathrm{O}$. La altitud es de 50 msnm, a 16 kilómetros de la ciudad de Guayaquil. Una condicion climática de temperatura media anual en época de lluvia de $35^{\circ} \mathrm{C}$ y en época seca de $18^{\circ} \mathrm{C}$. La precipitación media anual es entre 500 y 700 $\mathrm{mm}$. Está clasificado ecológicamente según el sistema de zonas de vida de Holdridge (1982) como un bosque seco Tropical (Tropical dry forest). Se realizaron cuatro salidas de reconocimiento de campo a la nueva ZRAL estableci- do con anterioridad en el plan de manejo de la Fundacion Pro bosque, BPCB donde se implementaría los nuevos senderos. Por medio de evaluación visual se elaboró un listado de los factores bióticos y abióticos existentes en el sitio de estudio (flora, fauna, clima y suelo, entre otros), el análisis histórico hasta la actualidad la nueva ZRAL del lugar, se determinó a través de una entrevista con el guardaparque más antiguo. Para la elaboración del inventario de atractivos turísticos de la nueva ZRAL se usó la metodología establecida por el Ministerio de Turismo (MINTUR, 2004) donde se clasifica los atractivos turísticos de acuerdo a categoría, tipo, subtipo. A su vez esta clasificación se divide en dos grupos: sitios naturales y de manifestación cultural. Cada lugar fue fotografiado con una Cámara Sony Dsc-hx200v Semi Profesional 60 x 18.2 Mpx 3d Hd. La jerarquización se realizó con un análisis individual de características intrínsecas y extrínsecas de cada atractivo, cada punto encontrado fue geo referenciado con las coordenadas geográficas de los atractivos identificados. Luego se ingresaron todos los datos al sistema de información geográfica (SIG) mediante el programa ArcView 8.3 y se estableció un mapa con la ubicación de los atractivos turísticos.

\section{RESULTADOS}

\section{Diagnóstico de la zona de estudio}

Los antecedentes históricos obtenidos de la entrevista al señor Perfecto Yagual o popularmente conocido como Don "Perfecto", dieron como antecedentes históricos que la mayor parte de los terrenos que ahora conforman el BPCB han sido parte de las antiguas haciendas ganaderas "Palobamba" y "Mapasingue". Según algunos de los más antiguos moradores del sector los terrenos de la antigua hacienda han sido destinados a la tala y explotación de madera de especies como madera negra (Tabebuia billbergii), cocobolo (Cynometra bauhiniifolia), laurel (Cordia alliodora), tillo blanco (Brosimun alicastrum), Fernán Sánchez (Triplaris cumingiana), entre otras, que son usados como durmientes del ferrocarril y como leña para los hornos de cal. Estos han proporcionado materia prima para una gran actividad económica centrada en la explotación, transporte y exportación de maderas. El fácil acceso a los bosques y la existencia de mano de obra especializada, 
posibilitaron el establecimiento de un importante astillero, el árbol especialmente más apreciado era guachapelí (Albizia guachapele) que hoy en día sigue siendo usado en la construcción de canoas y botes pequeños. Tiempo después se establecieron potreros de pasto para el ganado, y en la parte más alta se sembró gramineas como maíz. Comenzando la década de los 40 se realizó la explotación de piedra caliza en forma artesanal, donde la piedra caliza se la ponía en hornos con leña y se la dejaba a fuego vivo hasta por tres días consecutivos, se dejaba enfriar la piedra y se le añadia agua para que forme la cal, lo cual servía para hacer pastas dentales y pinturas, entre otros. Los antiguos hornos de cal en el centro de visitantes del BPCB son evidencia de la historia del área. Posteriormente se construyó una infraestructura para el uso del público por parte del dueño de los terrenos, la Cemento Nacional S.A. en ese entonces, actualmente Grupo Holcim. Con el asesoramiento de varios técnicos impulsó la reconstrucción de unos antiguos hornos de cal, la construcción de un anfiteatro y senderos naturales, a más de una cabaña ecológica que ofrece hospedaje para 6 personas, una zona de camping con capacidad para 40 personas. Adicional a todo esto también hay un bar donde se puede comprar refrigerios o comidas rápidas.

\section{Descripción de los senderos existentes en el BPCB}

En el BPCB hay tres senderos guiados y tres auto guiados, los cuales tienen diferentes tiempos de duración, así como distancia de recorrido, lo cual depende de la topografía del terreno (inclinación o declinación) del sendero (Tabla 1).

\section{TABLA 1. Características de los senderos presentes en el BPCB.}

\begin{tabular}{lccc}
\hline Senderos & Tipos & $\begin{array}{c}\text { Tiempo de recorrido } \\
\text { (minutos) }\end{array}$ & $\begin{array}{c}\text { Distancia } \\
(\mathbf{m})\end{array}$ \\
Canoa & Guiados & 90 & 1.177 \\
Buena vista & Guiados & 60 & 1.274 \\
Buena vista Corto & Guiados & 90 & 2.588 \\
Buena vista Largo & Guiados & 270 & 5.096 \\
Avenida las aves & Auto guiados & 30 & 490 \\
Arboles gigantes & Auto guiados & 20 & 500 \\
Etnobotánico & Auto guiados & 30 & 610
\end{tabular}

Fuente: Con guía ida y venida auto guiado: sin guía por medio de señaléticas.

\section{Senderos guiados}

El sendero canoa se encuentra en buen estado ya que constantemente ha sido mantenido. Es el de más fácil acceso ya que posee una duración de una hora y media de recorrido y una distancia de 1.177 metros, su principal atractivo es la quebrada Canoa, la cual durante la época de lluvias permanece con abundante agua, junto con la vegetación abundante forman hermosos paisajes. Cuenta con infraestructura que facilita el acceso y disfrute del recorrido, tales como un puente rústico que atraviesa la quebrada Canoa, un mirador, escaleras de piedras y pasamanos. Como sistema de señalización, existen dos indicaciones de orientación en el sendero Canoa, la primera está al inicio del recorrido donde se indica el nombre del sendero, tiempo de recorrido y distancia a recorrer y la otra señal está en un árbol de ceibo indicando como normativa de conservación que no se puede escribir sobre los árboles. El sendero Buenavista se encuentra ligeramente deteriorado ya que ha sufrido la intervención de las manos enemigas del hombre como es el caso de los cazadores de animales que ingresan a este recorrido a cazar venados de cola blanca (Odocoileus peruvianus) y cuchuchos (Nasua nasua). Este sendero tiene una hora inicial de caminata, luego de esto el turista llega a una $(Y)$ donde debe elegir el recorrido en Buenavista corta con 2.588 metros de longitud y con un tiempo promedio 
de recorrido de una hora y media, mientras que el sendero Buenavista Largo tiene una longitud de 5.096 metros con un tiempo promedio de recorrido de tres horas y media. La infraestructura de apoyo la constituyen dos miradores y escaleras naturales formadas a base de la piedra caliza existente en la zona.

Los dos senderos llegan hasta la parte alta de la montaña. La diferencia entre éstos es la distancia de recorrido. Se pueden observar o escuchar una gran cantidad de aves como trogones (Trogon mesurus) y chachalacas (Ortalis erythroptera). También a veces se ven mamíferos como los sahinos (Pecari tajacu), guatusas (Agouti paca), venados de cola blanca (Odocoileus peruvianus), cuchuchos (Nasua nasua), cabeza de mate (Eira barbara) y monos aulladores (Alouatta palliata).

Como sistema de señalizacion se tiene una señal de orientación que se encuentra al inicio del sendero, la cual indica el nombre del sendero, tiempo y distancia a recorrer; también hay cinco señales interpretativas que van ayudar a los guías o a los turistas del BPCB, los cuales se ubican cada 600 y 1.000 metros aproximadamente.

\section{Senderos autoguiados}

Sendero Avenida de las Aves se encuentra a 200 metros de la puerta principal, es un sendero lineal que tiene 490 metros de distancia y el tiempo de recorrido depende de las personas que lo están transitando, pue tiene instalado sillas de bambú en dos lugares de descanso y se han colocado letreros interpretativos con un sistema de preguntas sobre las aves de Cerro Blanco. Este sendero tiene la intención de hacer más dinámica la caminata de los turistas que visitan el bosque hacia el centro de visitantes, ya que pueden caminar por éste y así evitar un poco el sol y el polvo, generado cuando se camina por la vía principal lastrada del BPCB y en ciertos lugares sin cubierta vegetal.

El Sendero de Árboles Gigantes tiene una distancia de 500 metros. El tiempo promedio de recorrido es de 20 minutos y está provisto de letreros interpretativos acerca de flora y fauna del bosque seco tropical, es un sendero que está ubicado entre árboles de ceibo (Ceiba trichistandra), en cuanto a la fauna se puede observar Caciques (Cacicus cela), quen-quen (Cyanocorax mystacalis), garrapateros (Crotophaga sulcirostris), carpinteros (Campephilus guayaquilensis), holleros (Furnarius cinnamomeus) y una gran cantidad de huellas de venados (Odocoileus peruvianus), cabeza de mate (Eira barbara).

El Sendero Etnobótanico fue designado con ese nombre gracias a la gran presencia de plantas silvestres de uso medicinal. Su entrada se encuentra en el área de camping, este sendero es lineal y conduce al aviario del BPCB, tiene un tiempo de caminata de aproximadamente 30 minutos, con una distancia de 610 metros. A lo largo del recorrido se encuentran letreros que brindan información sobre el uso medicinal que las personas han atribuido a estas especies arbóreas, las especies vegetales que se han utilizado como recurso a interpretar son samán (Samanea saman), anona de montana (Capparis didymobotrys), guasmo (Guazuma ulmifolia), sasafraz (Zanthoxylum setolosum), y bototillo (Cochlosperum vitifolium).

\section{Flora presente en el BPCB}

Fueron registradas 514 especies vegetales presentes en la zona del BPCB; numéricamente las familias más predominantes son la Fabacea con 90 especies (18\%), seguida de las Asteraceas con 38 especies (7\%) y la Poacea de 28 especies (5\%), aunque existe poca presencia de géneros pertenecientes a las familia bombcacaea, en número se encuentran gran cantidad de árboles como Bombacopsis trinetensis (Ceibo macho) Cavanillesia plantanifolia E. (Pigio) Ceiba trichistandra E. (Ceibo) y Pseudobombax guayasense (Beldaco) los cuales son muy característicos de los bosques secos tropicales. Asi también, una de las especies usadas durante décadas son el Sapindus saponaria (Jaboncillo) perteneciente a la familia Sapindacea usado popularmente para lavar ropa y elaborar jabones, Guasuna ulmifolia (guasmo) de la familia de las Esterculacea, siendo esta especie muy típica de las zonas circundantes de la ciudad de Guayaquil y por ello existen barrios con ese nombre, Vitex gigantea (pechiche) perteneciente a las Verbenacea el cual se usa sus frutos para la elaboración de dulces y mermeladas (Tabla 2). 
TABLA 2. Flora presente en el BPCB.

\section{Acanthaceae}

Blechnum pyramidatum

Aphelandra glabrata (E)

Aphelandra guayasii (E)

Beloperone gutata

Carlowrigthia ecuadoriana (E)

Dicliptera multiflora

Dyschoriste ciliata

Elytraria imbricata

Justicia brandegea

Pseuderanthemum idroboi

Ruellia floribunda

Ruellia malacosperma

Sanchezia parviflora

Tetranium nervosum

Aphelandra glabrata (E)

Aphelandra guayasii (E)

Carlowrigthia ecuadoriana (E)

Dicliptera multiflora

Dyschoriste ciliata

Elytraria imbricata

Justicia brandegea

Pseuderanthemum idroboi

ANNONACEAE

Annona muricata L. (E)

Malmea lucida diles (E)

Raimondia quiduensis var. Latifolia

\section{ALIMATACEAE}

Echinodorus paniculatis

Sagitaria latifolia Willd

\section{BIGNONIACEAE}

Macfadyena unguis - cati (E)

Amphilophium ecuadorense

Amphilophium paniculatum

Anemopaegma chrysanthum

Anemopaegma puberulum

Arrabidaea candicans

Arrabidaea corallina

Arrabidaea pubescens

Crescentia cujete

Cydista decora

Spathodea campanulata

Jacaranda mimosifolia

Lundia corymbifera

Macfadyena unguis - cati (E)

Macranthisiphom longiflorus

Mansoa Hymenaea
Amaranthaceae

Alternanthera halimifolia

Alternanthera pubiflora

Alternanthera villosa

Amaranthus dubius

Amaranthus spinosus

Chamissoa altissima

Gomphrena globosa

Gomphrena serrata

AMARYLLIDACEA

Bomarea Mirb

Crinum erubenses

Crinum zelanicum (E)

Eurocrosia brachyandra

Eurocrosia stricklandii

Hippeastrum peniceum

Leptochiton quitoensis

ANACARDIACEAE

Mangifera indica

Mauria heterophylla

Spondias mombin

Spondias purpurea $L$.

ARACEAE

Dieffebachia sodiroi

Croat Monstera Adams (E)

Philodendron mexicanum

Philodendron Selloum

Pistia stratiotes

Syginium podophyllum

BOMBACACEAE

Bombacopsis trinetensis

Cavanillesia plantanifolia (E)

Ceiba trichistandra (E)

Eriotheca ruizzi

Matisia cordata

Ochroma pyramidale (E)

Pseudobombax guayasense

BORAGINACEAE

Cordia alliodora

Cordia collococca

Cordia hebeclada (E)

Cordia lutea (E)

Cordia macrantha

Cordia polyantha

Heliotropium curassavicum

Heliotropium indicum

Heliotropium rufipilum

\section{Arecaceae \\ Bactris setulosa}

APOCYNACEAE

Aspidosperma jaunachense(E)

Aspidosperma ulei

Catharanthus roseus

Fosteronia G.

Subsagittata Nerium oleander $L$.

Plumeria rubra

Pestronia mollis

Pestronia tomentosa

Rauvolfia tetraphylla

Stemmadenia obovatta

ASCLEPIADACEAE

Asclepias curassavica L.

Macroscepis hirsuta

Macroscepis urceolata

Marsdenia ecuadorensis

ASTERACEAE

Adenostemma platyphyllum

Baltimora recta (E)

Barnadesia lehmannii

Blumea viscosa

Brickellia diffusa

Chromolaena odorata

Chromolaena roserum

Condylidium iresinoides

Conyza bonariesis

Cyanthillium cinereum

Delilia biflora

Dendranthema indicum

Eclipta prostata

Emilia fosbergii

Enydra fluctuans

Erigeron $L$.

Eupatotium L. (E)

Isocarpha microcephala

Lagascea mollis Liabum (E)

Liabum eggersii

Lycoseris trinervis

Mikania cordifolia (E)

Mikania ecuadorensis

Pappobolus S.F.

Pluchea carolinensis

Pseudognoxys scabra

Schizoptera peduncularis

Senecio 
Paragonia pyramidata

Pithecoctenium crucigerum (E)

Tabebuia billbergii subsp. ampla

Tabebuia chrysantha Subsp. chrysantha

Tabebuia rosea

Tecoma castanifolia

Tecoma weberbaueriana

BROMELIACEAE

Pitcairnia unilateralis

Tillandsia complanata

Tillandsia flagellata

Tillandsia monadelpha

Tillandsia multiflora

Tillandsia triglochinoides

Tillandsia usneoide

Vriesea barclayana

\section{CHENOPODIACEAE}

Chenopodium ambrosioides

\section{COMMELINACEAE}

Callisia cordifolia

Commelina diffusa

Commelina coelestis

Commelina erecta

Commelina L.

Rhoeo spathacea

\section{CONVOLVULACEAE}

Turbina Raf.

Convolvulus nodiflorus

Cuscuta americana

Ipomoea asarifolia

Ipomoea carnea subsp. fistulosa

Ipomoea hederifolia $L$.

Ipomoea meyeri

Ipomoea nil

Ipomoea ophioides

Ipomoea trifida

Merremia aegyptia

Merremia umbellata

Operculina codonantha

Operculina hamiltonii

Turbina abutiloides

ERYTHROXYLACEAE

Erytroxylum patens

FABACEAE

Acacia macracantha
Tounerfotia maculata

Tounerfotia scabrida

Tounerfotia volubilis

\section{BURSERACEAE}

Bursera graveolens

\section{CACTACEAE}

Cereus cartwrightianus

Hylocereus polyrhizus

CAPPARACEAE

Capparis angulata (E)

Capparis ecuadorica

Capparis flexuosa subsp.

lanceolata

Capparis frondosa

Capparis heterophylla

Capparis petiolaris

Cleome parviflora subep.

psoralaefolia

Cleome pilosa

CHRYSOBALANACEAE

Licania Aubl.

COCHLOSPERMACEAE

Cochlospermum vitifolium

\section{CUCURBITACEAE}

Cayaponia A.

Citrillus lanatus

Cucumis dipsaceus

Cucumis L.

Cucurbita L.

Cucurbita moschata

Cyclanthera explodens

Echinopepon Naudin

Gurania Melothria hookeri

Momordica Charantia

DICHAPETALACEAE

Dichapetalum

asplundeanum(E)

Dichapetalum froesii

EUPHORBIACEAE

Acalypha diversifolia

Acalypha hispida

Acalypha subcastrata

Chamaesyce hirta

Chamaesyce hypericifolia

Chamaesyce lasiocarpa

Croton eggresii

Croton lechleri

Croton rivinifolius
Spilanthes Jacq.

Spilanthes ocymifolia

Struchium sparganophorum

Tridax procubens (E)

Trixis antimenorrhoea (E)

Verbesina eggersii

Verbesina Pentantha

Vernonanthura patens

Vernonia cinerea

Wedelia grandiflora

CARICACEAE

Carica papaya

Carica parviflora (E)

CECROPIACEAE

Cecropia litoralis (E)

CELASTRACEAE

Cecropia litoralis (E)

Maytenus ebenifolia (E)

Maytenus octogona

COMBRETACEAE

Combretum decadrum (E)

Combretum pavonii (E)

Terminalia valverdeae

CYPERACEAE

Cyperus alternifolius

Cyperus esculentus

Cyperus iria

Cyperus luzulae

Cyperus panamensis

Cyperus rotundus

Eleocharis geniculata

Rhynchospora Vahl

DIOSCOREACEAE

Dioscorea pilcomayensis

\section{EBENACEAE}

Diospyros inconstans

ELAEOCARPACEAE

Muntigia calabura

FLACOURTIACEAE

Casearia aculeata (E)

Prockia pentamera Xylosma

GESNERIACEAE

Episcia cupreata

HIPPOCRATEACEAEA

Elachyptera floribunda (E)

Salacia 
Acacia multipinnata

Acacia paniculata

Acacia riparia

Acacia tenuifolia

Aeschynomene americana

Aeschynomene sensitiva (E)

Aeschynomene tumbezensis

Albizia multiflora Albizia

Pistacifolia Alysicarpus vaginalis (E)

Bauhinia aculeata subsp. grandiflora (E)

Bauhinia seminariorioi (E)

Caesalpinia paipai

Caesalpinia pulcherrima

Canavalia brasiliensis

Canavalia rosea

Canavalia villosa

Cassia fistula

Cassia javanica (E)

Centrolobium ochroxylum

Centrosema plumieri

Centrosema pubescens

Centrosema virginianum

Chaetocalyx latisiliqua

Chamaescrita nictitans (E)

Clitoria andrei (E)

Clitoria Brachystegia

Clitoria ternatea

Cojoba rufescens

Courtesia caribaea

Crotalaria incana

Crotalaria pallida

Crotalaria pumila

Crotalaria retusa

Cynometra bauhiniifolia

Dalea cliffortiana

Desmodium distortum

Desmodiumpo eppigianum

Desmodium scorpiurus

Desmodium tortuosum

Entada polystachya

Erytrina fusca (E)

Erythrina velutina

Geoffroea spinosa (E)

Gliricidia brenningii

Gliricidia sepium Indigofera

Inga manabiensis (E)

Inga edulis

Inga sapindoides
Dalechampia scandens

Euphorbia graminea

Euphorbia heterophylla

Euphorbia L.

Euphorbia Lactea

Jatropha curcas

Manihot brachyloba

Margaritaria nobilis

Pedilanthus tithymaloides

Ricinus communis

Sapium laurifolium

\section{LILIACEAE}

Smilax lappacea

LOASACEAE

Mentzelia aspera $L$.

LOGANIACEAE

Buddleja americana

LORANTHACEAE

Phoradendron quadrangulare

Phthirusa retroflexa

Struthanthus chordocladus

MELASTOMATACEAE

Miconia gracilis

Miconia laevigata

MELIACEAE

Cedrela odorata

Guarea glabra

Trichilia elegans

Trichilia elegans subs. elegans

Trichilia hirta

MENISPERMACEAE

Cissampelos grandifolia

Cissampelos pareira

Disciphania ernstii (E)

Disciphania juliflora

NYCTAGINACEAE

Boerhavia coccinea

Boerhavia difusa

Boerhavia erecta

Bougainvillea Comm.

Cryptocarpus pyriformis

Mirabilis violaceae

Nee sp.

Pisonia aculeata

Pisonia macranthocarpa

\section{OLACACEAE}

Schoepfia cshreberi

Ximenia americana

\section{LAMIACEAE}

Hyptis pectinata

Scutellaria agrestis

LAURACEAE

Nectandra martinicensis

Nectandra purpurea

Ocotea veraguensis

LECYTHIDACEAE

Gustavia angustifolia (E)

Gustavia pubescens (E)

MALPIGHIACEAEA

Bunchosia Rich Hiraea

Malpighia retusa

Stigmaphyllon ellipticum (E)

Stigmaphyllon nudiflorum

Stigmaphyllon ovatum

MALVACEAE

Abutilon dispermun (E)

Abutilon multiflorum (E)

Abutilon reflexum (E)

Abutilon virgatum

Bastardia viscosa

Gossypium barbadense

Malachra humilis

Malvastrum tomentosum

Malvaviscus concinnus

Pavonia mollis

Sida glabra

Sida hederifolia

Sida javensis

Sida rhombifolia

Sida spinosa

\section{MORACEAE}

Brosimun alicastrum subs. bolivarense

Brosimun alicastrum

Dorstenia contrajerva

Ficus citrifolia

Ficus maxima

Ficus gomelleira

Ficus jacobii

Ficus microcarpa

Ficus obtusifolia

Ficus velutina (E)

Sorocea sprucei

MUSACEAE

Heliconia hirsuta

Heliconia psittacorum

MYRSINACEAE 
Leucaena leucocephala

Leucaena trichodes

Lonchocarpus atropurpureus

Machaerium Capote (E)

Machaerium millei

Macroptilium lathyroides

Macroptilium longepedunculatum

Macroptilium Benth

Mimosa acantholoba

Mimosa albida

Mimosa debilis (E)

Mimosa debilis var. aequatoriana

Mimosa pigra

Myrotylon peruiferum

Mucuna pruriens

Neptunia oleracea

Neptunia plena

Pachyrhizus palmatibolus

Parkinsonia aculeata

Phaseolus lunatus var. lunatus

Piscidia carthagenensis

Pithecellobium excelsum

Poiretia punctata

Prosopis juliflora

Pseudosamanea guachapele

Rhynchosia calycosa

Rhynchosia minima

Samanea saman

Samanea tubulosa

Senna bicapsularis (E)

Senna bicapsularis var. augusti

Senna bicapsularis var. bicapsularis

Senna mollissima

Senna mollissima var. mollissima

Senna obtusifolia

Senna oxyphylla (E)

Senna oxyphylla var. hartwegii

Senna pilifera

Senna pistaciifolia

Senna pistaciifolia var. picta

Senna siamea

Sesbaina emerus

Teramnus uncinatus

Teramnus volubilis

Vigna luteola

Vigna peduncularis

Vigna unguiculata

Zapoteca caracasana

\section{ONAGRACEAE}

Ludwigia leptocarpa

Ludwigia octovalvis

OPILIACEAE

Agonandra

OXALIDACEAE

Oxalis jasminifolia

Oxalis microcarpa

\section{PASSIFLORACEAE}

Passiflora edulis

Passiflora filipes

Passiflora foetida (E)

Passiflora macrophylla

Passiflora morifolia

Passiflora punctata

Passiflora resticulata

Passiflora rubra

Passiflora rubra Vell (E)

Passiflora sprucei

Passiflora suberosa (E)

Passiflora tenella

PLUMBAGINACEAE

Plumbago scandens

\section{POACEAE}

Cenchrus brownii

Chloris inflata

Chloris radiata

Dactyloctenium aegyptium

Dichanthium aristatum

Digitaria bicornis

Echinochloa colona

Eleusine indica

Eragrostis ciliaris

Eriochloa pacifica

Eriochloa stevensii

Ichnanthus oplismenoides

Lasiacis ruscifolia

Lasiacis sorghoidea

Leersia hexandra

Leptochloa virgata

Olyra latifolia

Oplismenus hirtellus

Panicum hirticaule

Panicum maximun

Paspalum cognatissimum

Paspalum macropodium

Paspalum racemosum

Pennisetum occidentale
Ardisia Geissanthus

MYRTACEAE

Eucalyptus alba

Eucalyptus camaldulensis

Eugenia florida Myrcia (E)

Psidium aff acutangulum

ORCHIDACEAE

Aspasia psitticina

Brassavola grandiflora (E)

Cattleya maxima (E)

Cycnoches lehmannii (E)

Dimerandra rimbachii (E)

Encyclia aspera (E)

Epidedrum bracteolatum (E)

Lockhartia serra

Notylia replicata (E)

Oncidium planilabre

Oncidium Sw. (E)

Stelis Sw.

\section{PHYTOLACCACEAE}

Achatocarpus pubescens (E)

Hilleria latifolia

Hilleria secunda

Petiveria alliacea

Trichostigma octandrum

PIPERACEAE

Peperomia Piper amalago

Piper dilatatum

Piper eriopodon

Piper marginatum

Piper peltatum

Piper sancti - felicis

Piper tuberculatum

PTERIDOPHYTA

Acrostichum danaeifolium

Adiantum alarconianum

Adiantum concinnum

Adiantum henslovianum

Adiantum I.

Adiantum petiolatum

Adiantum raddianum

Adiantum tetraphyllum

Azolla caroliniana

Blechnum occidentale

Blechnum serrulatum

Lygodium venustum

Marsilea ancylopoda

Nephrolepis cordifolia 
Zapoteca caracasana subs. weberbaueri Zapoteca portoricensis subs. flavida Zornia thymifolia

PUNICACEAE

Punica granatum

POLYGALACEAE

Polygala platycarpa

Securidaca coriacea

POLYGONACEAE

Antigonon leptopus

Coccoloba mollis

Coccoloba obovata

Triplaris cumingiana

PORTULACACEAE

Portulaca umbraticola

Talinum paniculatum

RHAMNACEAE

Scutia spicata

Ziziphus thyrsiflora

RHIZOPHORACEAE

Rhizophora harrisonii

ROSACEAE

Rubus $L$.

\section{RUTACEAE}

Citrus L. (E)

Erythrochiton giganteus

Zanthoxylum fagara

Zanthoxylum setulosum

\section{SAPOTACEAE}

Chrysophyllum argenteum subsp.

panamense

Chrysophyllum caimito Pouteria Aubl (E)

Pradosia montana

\section{SCROPHULARIACEAE}

Scoparia dulcis

Stemodia durantifolia

VERBENACEAE

Aegiphila glomerata (E)

Aloysia dodsoniorum

Avicennia germinans (E)

Citharexylum quitense

Clerodendrum molle

Cornutia microcalycina

Cornutia pyramidata
Pennisetum peruvianum

Rhynchelytrum repens

Sporobolus tenuissimus

Syntherisma aequatoriensis

RUBIACEAE

Alseis eggersii (E)

Alseis peruviana (E)

Chiococca alba

Coffea arabica

Coutarea hexandra

Ixora chinensis

Ixora finlaysoniana Palicourea

Aubl

Psychotria horizontalis

Psychotria microdon

Psychotria tenuifolia

Randia aculeata

Randia espinosa

Simira ecuadorensis

SOLANACEAE

Acnistus arborescens

Acnistus frutescens

Browallia americana

Browallia elata

Browallia viscosa

Capsicum rhomboideum

Cestrum auriculatum

Exodeconus maritimus (E)

Lycopersicon glandulosum

Physalis pubescens

Solanum candidum

Solanum caripense

Solanum confertiseriatum

Solanum hazenii

Solanum rudepannum

Solanum umbellatum

STERCULIACEAE

Ayenia aliculata

Byttneria aculeata

Byttneria catalpaefolia

Byttneria glabrescens

Byttneria parviflora

Guazuna ulmifolia

Melochia Iupulina
Nephrolepis exaltata

Niphidium crassifolium

Pityrogramma calomelanos

Polypodium bombycinum

Selaginella silvestris

Thelypteris hispidula var.

versicolor

Thelypteris patens var.

scabriuscula

Thelypteris serrata

SABIACEAE

Meliosma herbertii

SANTALACEAE

Acanthosyris glabrata (E)

\section{SAPINDACEAE}

Allophylus racemosus subsp.

racemosus

Cardiospermun halicacabum

Cupania americana subs. latifolia

Cupania cinerea

Paullinia buricana

Paullinia fuscescens

Paullinia serjaniaefolia (E)

Paullinia tumbesensis

Sapindus saponaria

Serjania brevipes

Serjania grammatophora

Serjania longipes

Serjania mucronulata

Serjania rhombea Thinouia

THEOPHRASTACEAE

Clavija pungens (E)

Jacquinia pubescens (E)

\section{TILIACEAE}

Corchorus aestuans

Corchorus hirtus

Corchorus orinocensis

Triumfetta bogotensis

Triumfetta grandiflora

Triumfetta semitriloba

ULMACEAE

Celtis iguaneae

Trema micrantha

URTICACEAE

Bohemeria Pilea microphylla

Urera baccifera 


\author{
Lantana cujabensis \\ Lantana glutinosa \\ Lantana scabiosiflora (E) \\ Lantana svensonii \\ Lippia alba \\ Petrea volubilis \\ Phyla strigulosa \\ Priva lappulacea (E) \\ Vitex gigantea
}

\author{
TROPAEOLACEAE \\ Tropaeolum L. \\ TURNERACEAE \\ Turnera pumilea \\ TYPHACEAE \\ Typha domingensis \\ VITACEAE \\ Cissus sicyoides \\ Vitis tiliifolia
}

\author{
VIOLACEAE \\ Hybanthus attenuatus \\ Rinorea deflexa \\ Rinorea pubiflora \\ Rinorea viridifolia
}

ZINGIBERACEAE

Alpinia purpurata

Costus guanaiensis var. tarmicus

\section{Fauna presente en el BPCB}

Con respecto a la fauna se registraron 53 especies de mamíferos, de los cuales 24 especies pertenecen al Orden Chiroptera (Tabla 3). Durante las diferentes salidas de campo se pudo observar e incluso ver pisadas de venados (Mazama americana y Odocoileus peruvianus), armadillo común (Dasypus novemcinctus), monos aulladores (Alouatta palliata), micos (Cebus albifrons), guatusa (Dasyprocta punctata), se evidencia la presencia de dos especies endemicas de ardillas, sabanera de Guayaquil (Sciurus stramineus) y ardilla rojiza de occidente (Sciurus granatensis), siendo estas especies las más comunes de observar en las salidas. La presencia de "pozas" de agua permanentemente que se forman durante la estación seca (julio -diciembre) en las quebradas, son importantes para muchas especies de mamíferos y otros animales, especialmente los murciélagos (Glosso phaginac, Sturnira sp. y Artibeus sp.) que frecuentan las quebradas en búsqueda de agua y comida. Existe el registro de 219 especies de aves de las cuales 43 son endémicos de la zona de Endemismo Tumbesino del suroeste del Ecuador, noroeste del Perú,

Adicionalmente, en cuatro áreas protegidas existentes a nivel nacional, éstas albergan doce especies de aves globalmente amenazadas. El BPCB tiene nueve, tales como el Gavilán dorsigris (Leucopternis occidentalis), paloma vientriocracea (Leptotila ochraceiventris), jilguero azafranado (Carduelis siemiradzkii), colaespina colanegra (Synallaxis tithys), rascahojas capuchirrufa (Hylocryptus erythrocephalus), mosquerito pechigris (Lathrotriccus griseipectus), mosquero real (Onychorhynchus occidentalis), Chachalaca cabecirrufa (Ortalis erythroptera) y papagayo de Guayaquil (Ara ambiguus guayaquilensis). Un aspecto relevante de la avifauna del sector es la diversidad de es- pecies de aves rapaces, incluso 15 especies de gavilanes y elanios, asi también seis especies de halcones.

La herpetofauna marca una determinada importancia ya que solo en anfibios se pueden observar cuatro grupos principales; las ranas de árbol de las familias Hylidae y Leptodactylidae que se encuentran generalmente en la vegetación arbórea y arbustiva y/o cerca de pozas o riachuelos, generalmente son de actividad nocturna, especies características de esta zona son Trachycephalus jordani y Leptodactylus labrosu. Además se encontró el género Physalaemus (ranas bullangueras), que son ranas que viven asociadas a pozas pequeñas; sapos terrestres de la familia Bufonidae, los más comunes son el Bufo marinus y Bufo margaritifer que es más pequeño que el anterior y habita en la hojarasca. Las ranas de la familia Dendrobatidae del género Colostethus son de coloración generalmente café oscura y son poco tóxicos, existen por lo menos dos especies en esta familia, es común observarlas cantando durante el día en las inmediaciones de la quebrada del sendero Canoa.

En cuanto a reptiles existen cuatro familias de importancia donde los Boidae se caracterizan por su considerable tamaño, estos pueden llegar a medir hasta 4,5 metros de largo, se les llama boas o matacaballos (Boa constrictor imperator). Los Colubridae son culebras comunes vulgarmente llamadas como lisas, sobrecamas y cordoncillos, las cuales presentan coloraciones entre verde y café, algunas pueden ser negras como la chonta (Clelia clelia), se apreciaron otras de colores vivos consideradas como falsas corales; otra familia temida por su veneno hemolítico son las viperidae de las cuales se presenta en el área de estudio la Víbora o nombrada también como "equis" 
(Bothrops asper), se avistó vivoras dentro de las consideradas altamente venenosas algunas como Elapidae, representadas por las corales que poseen veneno neurotóxico. Por último se avisoró reptiles de la familia de los Saurios de tres grupos, las lagartijas arborícolas que incluyen especies como la iguana verde (Iguana iguana), el falso camaleón (Polychrus gutturosus spurelli) y lagartijas arbóreas (Anolis gracilipes), las lagartijas terrestres como las lagartijas de vistosos colores (Ameiva edracantha y Ameiva septemlineata) que se encuentran en los bordes de los caminos, y por ultimo las lagartijas trepadoras (Geckonidae), tenemos especies representativas como salamanquesa común (Phyllodactylus reissii) y Gonatodes caudiscutatus.

TABLA 3. Mamíferos y aves presentes en el BPCB.

\begin{tabular}{|c|c|c|}
\hline Agoutidae & Dasyproctidae & Emballonuridae \\
\hline \multicolumn{3}{|c|}{ Mamíferos } \\
\hline Agouti paca & Dasyprocta punctata & Saccopterix bilineata \\
\hline CANIDAE & DASYPODIDAE & ERENTHIZONTIDAE \\
\hline Pseudolopex sechurae & Dasypus novemcinctus & Coendou rothschildi \\
\hline CEBIDAE & DIDELPHIDAE & LEOPARIDAE \\
\hline Allouatta palliatta & Didelphis albiventris & Sylvilagus brasilensis \\
\hline Cebus albifrons & Didelphis marsupialis & MURIDAE \\
\hline CERVIDAE & Philander opossum & Oryzomys caliginosu \\
\hline Manzama americana & Marmosa sp. & Oryzomys xantheolus \\
\hline Odocoileus virginianus & EDENTATA & MUSTELIDAE \\
\hline ECHIMYDAE & Choloepus haffmani & Eira barbara \\
\hline Proechimys sp. & Bradypus variegatus & Galictis vittata \\
\hline Proechimys decumanus & MOLOSSIDAE & Mustela frenata \\
\hline PHYLLOSTOMIDAE & Molossus molossus & NOCTILIONIDAE \\
\hline Carollia castanea & MYRMECOPHOGIDAE & Noctilio leporinus \\
\hline Carollia perspicillata & Tamandua mexicana & SCIURIDAE \\
\hline Desmodus rotundus & Myrmecophaga tetradactyla & Sciurus stramineus \\
\hline Uroderma bilobatum & PROCYONIDAE & Sciurus granatensis \\
\hline Artibeus sp. & Nasua narica & VESPERTILIONIDAE \\
\hline Artibeus litoratus & Procyon cancrivorus & Myotis nigricans \\
\hline Artibeus jamaicensis & Potos flavus & Myotis riparius \\
\hline Atibeus fraterculus & TAYASSUIDAE & Lasiurus borealis \\
\hline Chiroderma villosum & Pecari tajacu & \\
\hline Platyrrhinus helleri & FELIDAE & \\
\hline Enchisthenes hartii & Puma concolor & \\
\hline Lonchophylla robusta & Leopardus pardalis & \\
\hline Sturnira spp. & Leopardus wiedii & \\
\hline Sturnira lilium & Herpailurus yagouarundi & \\
\hline Phyllostomus hastatus & Panthera onca & \\
\hline \multirow{2}{*}{\multicolumn{3}{|c|}{$\begin{array}{l}\text { Lonchophylla spp. } \\
\text { Glossophaga soricina }\end{array}$}} \\
\hline & & \\
\hline \multicolumn{3}{|c|}{ Aves } \\
\hline ACCIPITRIDAE & ALCEDINIDAE & APODIDAE \\
\hline Gampsonyx swainsonii & Ceryle torquata & Chaetura brachyura \\
\hline Ictinia plumbea & Chloroceryle americana & Chaetura cinereiventris \\
\hline Rostrhamus sociabilis & Chloroceryle aenea & Streptoprocne zonaris \\
\hline
\end{tabular}


Harpagus bidentatus

Chondrohierax uncinatus

Asturina nitida

BUCCONIDAE

Notharchus macrorhynchos

\section{CATHARTIDAE}

Cathartes aura

Coragyps atratus

Sarcoramphus papa

CARDINALINAE

Saltator maximus

Saltator albicolis

Pheucticus chrysogaster

\section{COTINGIDAE}

Pachyramphus homochrous

Pachyramphus albogriseus

Pachyramphus cinnamomeus

Pachyramphus spodiurus

CUCULIDAE

Crotophaga ani

Crotophaga sulcirostris

Tapera naevia

Piaya cayana

Coccyzus lansbergi

Coccyzus americanus

FORMICARIIDAE

Taraba major

Sakesphorus bernardi

Dysithamnus mentalis

Pyriglena pacifica

Grallaria watkinsi

HIRUNDINIDAE

Notiochelidon cyanoleuca

Progne chalybea

Stelgidopteryx ruficollis

ICTERIDAE

Cacicus cela

Subfamilia ICTERINAE

Dives warszewiczi

Molothrus bonariensis

Sturnella bellicosa

Icterus mesomelas

Icterus graceannae

Amblycercus holosericeus

Scaphidura oryzivora

Psarocolius angustifrons

\section{ANATIDAE}

Dendrocygna autumnalis

CAPRIMULGIDAE

Nyctidromus albicollis

Caprimulgus anthony

Chordeiles acutipennis

CARDUELINAE

Carduelis siemiradzkii

CHARADRIIDAE

Acittis macularia

Chanadrius vociferus

Tringa melanoleuca

COEREBINAE

Coereba flaveola

CORVIDAE

Cyanocorax mystacalis

CRACIDAE

Ortalis erythroptera

Penelope purpurescens

EMBERIZINAE

Sporophila americana

Sporophila peruviana

Sporophila telasco

Sicalis flaveola

Rhodospingus cruentus

Volatinia jacarina

Arremon abeillei

FREGATIDAE

Fregata magnificens

Familia FURNARIIDAE

Subfamilia

DENDROCOLAPTINAE

Dendrocinela fuliginosa

Campylorhamphus trochilirostris

Lepidocolaptes souleyetii

Sittasomus griseicapillus

MIMIDAE

Mimus longicaudatus

MOMOTIDAE

Momotus momota

PANDIONIDAE

Pandion haliaetus

PELECANIDAE

Pelecanus occidentalis

PHALACROCORACIDAE

Phalacrocorax olivaceus

PHASIANIDAE

Odontophorus erythrops
Panyytila cavennenses

ARDEIDAE

Egretta alba

Ardea cocoi

Egretta thula

Bubulcus ibis

Butorides striatus

\section{COLUMBIDAE}

Claravis pretiosa

Columba cayennensis

Columba livia

Columbina buckleyi

Columbina cruziana

Zenaida asiatica

Leptotila verreauxi

Leptotila ochraceventris

Leptotila pallida

EMBERIZIDAE

Hemithraupis guira

Conothraupis speculigera

FALCONIDAE

Polyborus plancus

Herpetotheres cachinnans

Micrastur semitorquatus

Micrastur ruficollis

Daptrius americanus

Falco rufigularis

Falco peregrinus

Falco columbarius

Falco sparverius

\section{HELIORNITHIDAE}

Heliornis fulica

Mycteria americana

NYCTIBIIDAE

Nyctibius griseus

PARULIDAE

Parula pitiayumi

Setophaga ruticilla

Seiurus noveboracensis

Basileuterus fraseri

Geothlypis aequinoctialis

Geothlypis auricularis

\section{PSITTACIDAE}

Amazona autumnalis lilacina

Aratinga erythrogenys

Brotogeris pyrrhopteris 
Dolichonyx oryzivorus

Familia TYRANNIDAE

\section{SubfamiliaTYRANNINAE}

Muscigralla brevicauda

Tyrannus savana

Megarhynchus pitangua

Myiodynastes maculatus

Myiodynastes bairdii

Myiozetetes cayanensis

Myiarchus phaeocephalus

Myiarchus tuberculifer

Lathroticcus griseipectus

Myiophobus fasciatus

Onycorhynchus occidentalis

Todirostrum cinereum

Euscarthmus meloryphus

Myiopagis subplacens

Elaenie flavogaster

Phaeomyias murina

Camptostora obsoletum

Leptopogon superciliaris

Pipromorpha oleaginea

Conopias parva

Lophotriccus pileatus

Tolmomyias sulphurescens

Contopus cinereus

Fluvicola nengeta

Myiozetetes similis

Pyrocephalus rubinus

Tyrannus melancholicus

Tyrannus niveigularis

Piranga flava

Piranga rubla

Myiobius atricaudus

\section{PODICIPEDIDAE}

Tachydaptus dominicus

RALLIDAE

Aramides axillaris

Gallinula chloropus

THRAUPIDAE

Euphonia saturata

Euphonialaniirostris

Thraupis episcopus

Tachyphonus luctuosus

Hemithrampis guira

Piranga flava

Piranga rubra

TROGLODYTIDAE

Campylorhynchus fasciatus

Thryothorus superciliaris

Troglodytes aedon

Thryothorussclareti

TYTONIDAE

Tyto alba

VIREONIDAE

Cyclarhis gujanensis

Vireo olivaceus

Hylophilus minor

TROCHILIDAE

Phathornis superciliosus

Phaethornis longuemareus

Anthracothorax nigricollis

Heliomaster longirostris

Myrmia micrura

Amazilia amazilia

Chlorostilbon

Damophila julie

Acestrura berlepschi
Forpus coelestis

Ara ambiguus guayaquilensis

Pionus chalcopterus

RHINOCRYPTIDAE

Melanopareia elegans

STRIGIDAE

Otus roboratus

Lophostrix cristata

Glaucidium peruanum

Pulsatrix perspicillata

Ciccaba nigrolineata

Glaucidium brasilianum

TROGONIDAE

Trogon violaceus

Trogon melanurus

TURDIDAE

Catharus ustulatus

Turdus maculirostris

Turdus reevei

TINAMIDAE

Crypturellus transfaciatus

PICIDAE

Picumnus sclateri

Piculus ribiginosus

Melanerpes pucherani

Campephilus guayaquilensis

Veniliornis kirkii

Veniliornis callonotus

Dryocopus lineatus

\section{Inventario de los atractivos}

\section{turísticos de la nueva ZRAL del BPCB}

Se realizó el inventario en la nueva ZRAL donde se considerará la implementación de dos Senderos nuevos los cuales serán denominados Sendero "Buena Vista Dos" y Sendero "San Agustin". Ls atractivos pertenecientes a dichos senderos fueron georeferenciados y colocados en un mapa de referencia para su fácil acceso de ubicación a los turísticos (Figura 1). Todos los atractivos fueron cate- gorizados como sitios naturales, siendo el atractivo "Pared Rocosa" de tipo fenómeno geológico; éste se encuentra en el nuevo sendero "San Agustín" a 20 metros del centro de visitantes y está a 1,5 km de la carretera principal vía a la costa. La Pared Rocosa tiene un largo aproximado de 50 metros de largo por 20 metros de alto, la pared es de color gris con ciertas partes blancas. Este es un punto 
para detallar la parte geológica predominante de la zona, en la actualidad no tiene ningun uso, pero en el futuro se pretende utilizar para practicar deportes como el rapel, ascenso y descenso en la montaña.

La "Quebrada San Agustín" es un atractivo de tipo Montaña, subtipo Desfiladero se encuentra a casi 200 metros del centro de visitantes, además está a $2 \mathrm{~km}$ de la carretera principal vía a la costa. Siempre hay la presencia de agua, por lo cual existe mucha humedad y también plantas de este tipo de hábitat como camachos, hongos y helechos. Dentro de la quebrada existen cangrejos de agua dulce, reptiles y anfibios, siendo este último origen de su uso principal ya que se realizan estudios constantes sobre anfibios. "La Quebrada" tiene 200 metros de largo y 10 metros de ancho en unas partes y en otros 18 metros (hasta donde se puede ingresar y medir).

El "Mirador Buenavista" es de tipo Montaña. Este mirador se encuentra en la parte alta del nuevo Sendero Largo, justamente cuando se empata con los antiguos senderos en el cual hay una vista formidable hacia los alrededores, donde se destaca uno de los pocos remanentes de manglar que se encuentra en el recinto de Puerto Hondo y la belleza paisajística que existe en sus alrededores, posee un espacio de 10 por 5 metros en el cual se puede ver la vegetación del manglar que se encuentra en el recinto "Puerto Hondo", las piscinas camaroneras y la represa de "Parque Lago".

Estos tres atractivos turísticos son de Jerarquia II, es decir, son atractivos con algún rasgo llamativo, capaz de interesar a visitantes de larga distancia, ya sea del mercado interno y receptivo, que hubiesen llegado a la zona por otras motivaciones turísticas o de motivar corrientes turísticas potenciales actuales para asi atraer al turismo de esparcimiento.

"Paisajes naturales de Bosque Seco Tropical" es un tipo Bosque de subtipo Seco Tropical. Estos paisajes se los puede observar durante el recorrido por cualquiera de los senderos, en especial desde las partes altas o desprovista de vegetación donde se logra tener una mayor visibilidad, donde se aprecie dentro de las 6.048 hectareas, árboles propios de estos bosques como el ceibo (Ceiba trichistrandra), pigio (Cavanillesia platanifolia), pepito colorado (Eritrina velutina), enredaderas o bejucos en general. Este atractivo es considerado de Jerarquía III, es decir, que posee rasgos excepcionales en un país, capaz de motivar una corriente actual o potencial de visitantes del mercado interno, y en menor porcentaje el internacional, ya sea por sí solos o en conjunto con otros atractivos contiguos (Tabla 4).

TABLA 4. Características de los atractivos turísticos del BPCB.

\begin{tabular}{|c|c|c|c|c|c|}
\hline \multirow{2}{*}{ Nombre atractivo } & \multirow{2}{*}{ Categoría } & \multirow{2}{*}{ Tipo } & \multicolumn{2}{|c|}{ Ubicación geográfica } & \multirow{2}{*}{ Jerarquización } \\
\hline & & & Latitud & Longitud & \\
\hline Pared rocosa & Sitio natural & $\begin{array}{l}\text { Fenómeno } \\
\text { geológico }\end{array}$ & $97^{\circ} 59^{\prime} 54.2^{\prime \prime S}$ & $60^{\circ} 99^{\prime} 34^{\prime \prime} \mathrm{W}$ & II \\
\hline Quebrada San Agustín & Sitio natural & Montaña & $97^{\circ} 59^{\prime} 60.5^{\prime \prime} \mathrm{S}$ & $60^{\circ} 99^{\prime} 67^{\prime \prime} \mathrm{W}$ & ॥ \\
\hline $\begin{array}{l}\text { Paisaje Natural del } \\
\text { Bosque Seco Tropical }\end{array}$ & Sitio natural & Bosques & $97^{\circ} 59^{\prime} 69.6$ " S & $60^{\circ} 99^{\prime} 63^{\prime \prime} \mathrm{W}$ & III \\
\hline Mirador Buenavista & Sitio natural & Montañas & $97^{\circ} 59^{\prime} 48.9^{\prime \prime} \mathrm{S}$ & $60^{\circ} 90^{\prime} 98 ” \mathrm{~W}$ & ॥ \\
\hline
\end{tabular}


FIGURA 1. Mapa de atractivos turísticos del sendero San Agustín.

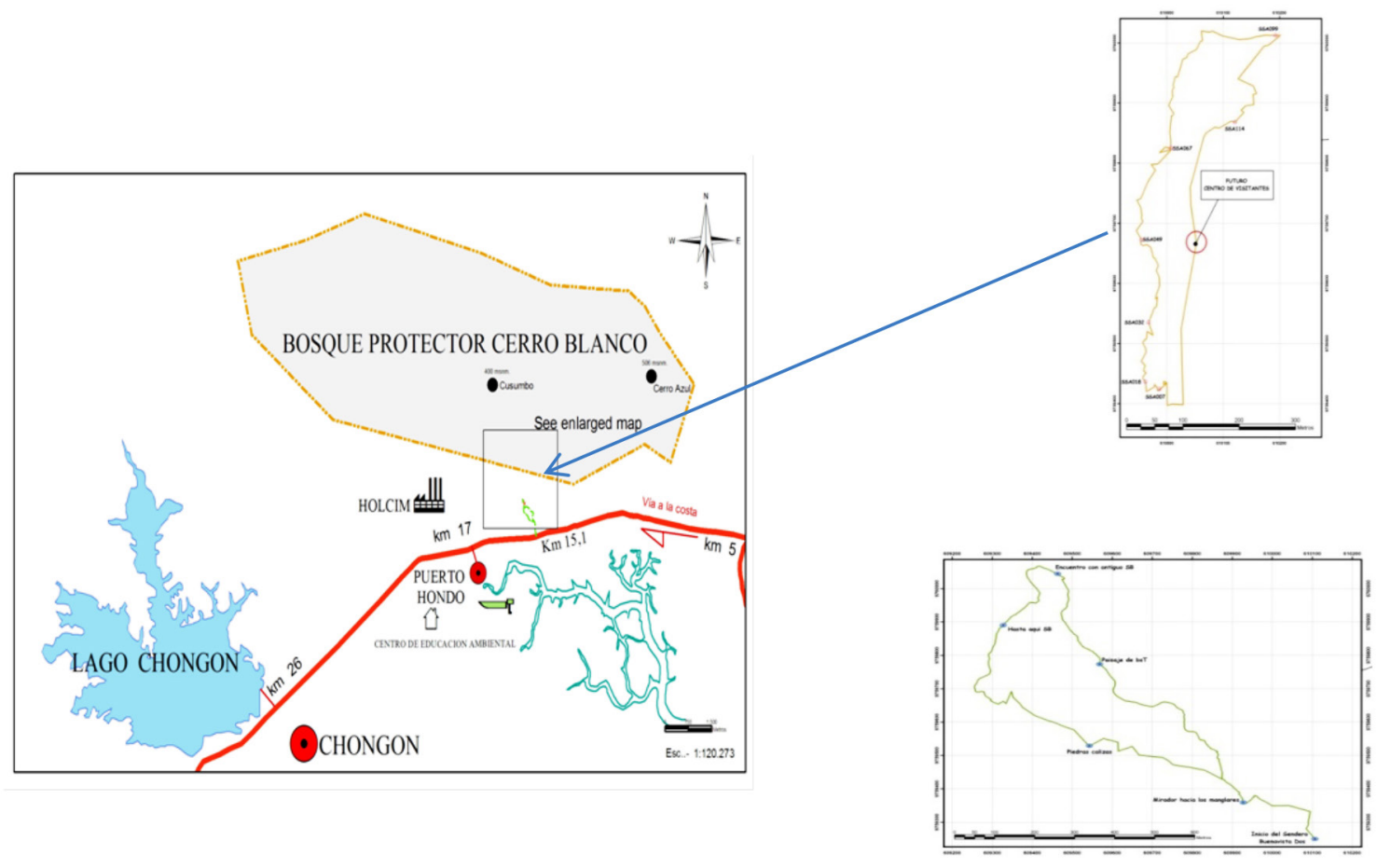

\section{CONCLUSIONES}

El Bosque Protector Cerro Blanco ofrece una de las mejores esperanzas para la conservación de un fragmento considerable del bosque seco tropical del Ecuador. Es bien conocido en la biología de conservación que, entre más grande sea el fragmento, más estable será ecológicamente y más segura es la protección que el fragmento pueda dar a las especies que lo habitan. Entonces la conservación del BPCB ofrece una de las mejores opciones para la sobrevivencia de tal vez solo 100 especies de plantas que son endémicas de la región del bosque seco tropical del Ecuador. Estas especies sin la protección brindada por el BPCB y pocas otras áreas en la región estarían amenazadas a la extinción global.

En uno de los primeros diagnósticos sobre el manejo del BPCB (Horstman, 1991) se propuso el establecimiento de una "zona limitada del ecoturismo" localizada en la boca de la quebrada Canoa en el km 15 vía a la costa. El sendero canoa se encuentra en buen estado ya que se ha tratado de mantener el número de visitantes de su capacidad de carga tanto para los senderos como para el área de visitantes del BPCB. Sin embargo, las detonaciones para extraer la piedra caliza de responsabilidad de Holcim Group es el principal factor que ha causado el desplazamiento de una gran variedad de especies de animales, tanto de aves como de mamíferos.

El Sendero de Árboles Gigantes tiene una distancia de 500 metros, el tiempo promedio de recorrido es de 20 minutos y está provisto de letreros interpretativos acerca de flora y fauna del bosque seco tropical, es un sendero que está ubicado entre árboles de ceibo (Ceiba trichistandra) y está diseñado para personas que no pueden o no desean caminar mucho, es relativamente plano y corto. 


\section{RECOMENDACIONES}

Realizar inventarios detallados de las especies de invertebrados en el BPCB a pesar de que aparentemente hay una gran riqueza, especialmente de especies acuáticas, como langostinos y cangrejos de agua dulce y de varias especies de lepidópteros, como la mariposa morpho. Esto porque no existe un documento oficial del registro de estas especies.

Se recomienda que los guardaparques del BPCB realicen con mayor frecuencia recorridos por toda el área para evitar la caza de los animales y esta actividad realizarla en conjunto con la Unidad de Protección de Medio Ambiente de la Policía Nacional (UPMA).

Los senderos tienen una longitud aceptable para que tengan el mismo flujo de visitantes y evitar el rechazo del sendero mas largo, por no caminar tanto.

\section{REFERENCIAS}

Angel, R. (2006). Memorias del X curso de Intérpretes Ambientales. Fauna del Bosque Seco Tropical, Ecuador.

Boo, E. (1992). La explosión del ecoturismo. Plan para el manejo y desarrollo. Editado por Mercedes Oteguiacha.

Carabajo, S., Horstman, E. (2005). Estrategia para el establecimiento de un corredor biológico para la conservación del Bosque Protector Cerro Blanco, con énfasis en el Guacamayo verde mayor (ara ambigua guayaquilensis).

Delgado, D. (2007). Diagnóstico potencial para el desarrollo del aviturismo en el Bosque Protector Cerro Blanco. Guayaquil-Ecuador.

Garcés, F. (1995). Guía metodológica para el desarrollo de proyectos de ecoturismo: Inventario, Diseño, Operación y Monitoreo, IDOM. Imprenta V\&O Gráficas. Quito - Ecuador.

González, W. (2006). Memorias del X curso de Intérpretes Ambientales. Geología del Bosque Seco Tropical, Ecuador.

Ham, S. (1992). Interpretación ambiental: una guía practica para la gente con grandes ideas y presupuestos pequeños. EE.UU.

Horstman, E. (1998). Plan de manejo del Bosque Protector Cerro Blanco.

MINTUR. (2004). Inventario de atractivos naturales y culturales. Ministerio de Turismo del Ecuador. 